Please do not remove this page

RMIT

UNIVERSITY

\title{
An efficient mobile Rayleigh fading channel simulator: A comparison with Clarke's Model
}

Shang, Lei; Hussain, Zahir; Harris, Richard

https://researchrepository.rmit.edu.au/esploro/outputs/9921858043401341/filesAndLinks?institution=61RMIT_INST\&index=null

Shang, L., Hussain, Z., \& Harris, R. (2004). An efficient mobile Rayleigh fading channel simulator: A comparison with Clarke's Model. IEEE TENCON 2004, 113-116.

https://doi.org/10.1109/TENCON.2004.1414720

Published Version: https://doi.org/10.1109/TENCON.2004.1414720

Repository homepage: https://researchrepository.rmit.edu.au

(c) 2004 IEEE. Personal use of this material is permitted. However, permission to reprint/republish this material for advertising or promotional purposes or for creating new collective works for resale or redistribution to servers or lists, or to reuse any copyrighted component of this work in other works must be obtained from the IEEE.

Downloaded On 2023/04/26 21:45:49 +1000 


\title{
AN EFFICIENT MOBILE RAYLEIGH FADING CHANNEL SIMULATOR: A COMPARISON WITH CLARKE'S MODEL
}

\author{
Lei Shang, Zahir M. Hussain, and Richard J. Harris \\ Centre for Advanced Technology in Telecommunications (CATT) \\ School of Electrical and Computer Engineering \\ RMIT University, Melbourne, Victoria 3000, Australia \\ Emails: s9612136@student.rmit.edu.au, zmhussain@ieee.org, richard@catt.rmit.edu.au
}

\begin{abstract}
This paper presents a computer model for Rayleigh fading channels. The simulated Rayleigh fading channel model is based on Smith's method and shows ease of implementation and greater mathematical tractability. We then investigate the level-crossing rate (LCR), the average duration of fades (ADF), the probability density function (PDF), the cumulative distribution function (CDF) and the autocorrelation functions (ACF) of this proposed model. The simulated results are verified against the analytical Clarke's channel model.
\end{abstract}

\section{INTRODUCTION}

Simulation models for fading channels are extremely important for the development, performance analysis, and test of modern wireless communication systems. The designed fading channel simulator should fit the desired statistical behavior with high precision before analyzing the performance of a new mobile communication system. Accuracy, efficiency, flexibility and ease of implementation are the challenging requirements in designing the simulation models [1].

The characteristic quantities describing the statistics of mobile fading channels are the probability density function (PDF), cumulative distribution function (CDF), the autocorrelation function (ACF), the levelcrossing rate (LCR) and the average duration of fades (ADF). The level-crossing rate (LCR) and average duration of fades (ADF) are useful for designing error control codes and diversity schemes to be used in mobile communication systems, since it becomes possible to relate the time rate of change of the received signal to the signal level and velocity of the mobile [2].

John I. Smith demonstrated a simple computer program in [3]. We modified this method and developed a new method which promises a greater mathematical tractability. Verification of this new method against the analytic fading channel model is thus necessary and a detailed analysis of the statistical characteristics of this new method is given.

The aim of this work is to design a fading channel simulator based on Smith's method and to analyze the LCR and ADF of this simulation model for

0-7803-8560-8/04/\$20.00(c)2004IEEE
Rayleigh fading channels. The results obtained will serve as the foothold for the further investigation of the recently proposed parabolic channel model [4], which will be analyzed in future works. Therefore, we first describe in Section II the stochastic reference model for the Rayleigh fading channel. In Section III, we briefly demonstrate the concept of zero-padding and up-sampling which will be exploited in the next section. Section IV presents the proposed Rayleigh fading simulator and the analysis of its statistical characteristics. The simulation results are compared with analytic results.

\section{DESCRIPTION OF THE ANALYTICAL MODEL}

The detailed derivation of Jakes Power spectral density or Clarke power spectral density can be found in [5]. We here review this derivation briefly and give some initial simulation results.

To derive the Jakes power spectral density, we follow these assumptions [6]:

1. The propagation of the electromagnetic waves takes place in the two-dimensional(horizontal) plane, and the receiver is located in the center of an isotropic scattering area.

2. The angles of arrival $\alpha$ of the waves arriving the receiving antenna are uniformly distributed in the interval $[-\pi, \pi)$.

3. The antenna radiation pattern of the receiving antenna is circular-symmetrical (omnidirectional antenna).

The probability density function of the angles of arrival $\alpha$ is thus given by

$$
p_{\alpha}=\left\{\begin{array}{lr}
\frac{1}{2 \pi}, & \alpha \in[-\pi, \pi), \\
0, & \text { elsewhere }
\end{array}\right.
$$

The Doppler frequencies can then be defined by

$$
f=f(\alpha):=f_{m} \cos (\alpha),
$$

where $f_{m}$ is the maximal Doppler frequency. Obviously $f$ is also a random variable. The probability density function of the Doppler frequencies $f$, denoted by 

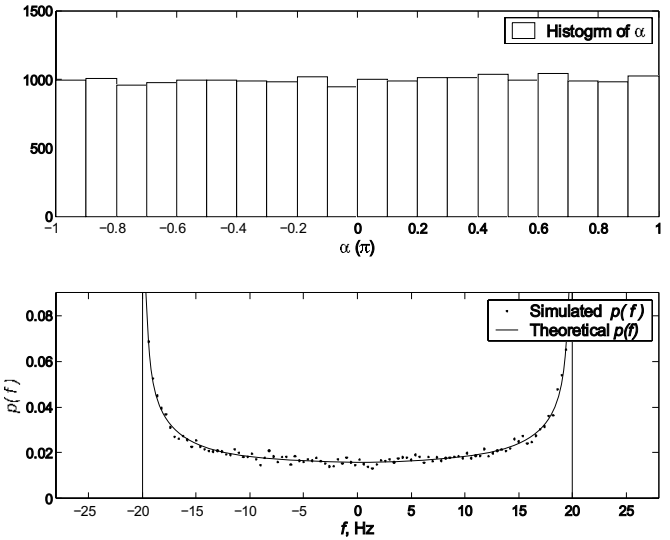

Fig. 1. Histogram of the angles of arrival $\alpha$ and the pdf of the Doppler frequencies $f$.

$p_{f}(f)$, can be given by [5]

$$
p_{f}(f)= \begin{cases}\frac{1}{\pi f_{m} \sqrt{1-\left(f / f_{m}\right)^{2}}}, & |f| \leq f_{m} \\ 0, & |f|>f_{m} .\end{cases}
$$

This function of random variables is simulated and shown in Fig. 1 with $f_{m}=20 \mathrm{~Hz}$.

The power spectral density $S_{\mu \mu}(f)$ of the scattered components $\mu(t)=\mu_{1}(t)+\mu_{2}(t)$, received at the receiving antenna, is obviously proportional to $p_{f}(f)$ of the Doppler frequencies. The following relation holds:

$$
S_{u u}(f) d f \sim p_{f}(f) d f,
$$

The relation between the probability density function and the power spectrum density is thus established. Further investigation which takes Eq.(3) into account will lead into the Jakes Power spectral density or Clarke power spectral density:

$$
S_{u u}(f)= \begin{cases}\frac{2 \sigma_{0}^{2}}{\pi f_{m} \sqrt{1-\left(f / f_{m}\right)^{2}}}, & |f| \leq f_{m}, \\ 0, & |f|>f_{m} .\end{cases}
$$

where $\int_{-\infty}^{\infty} S_{u u}(f) d f=2 \sigma_{0}^{2}$, and $2 \sigma_{0}^{2}$ is the power of the scattered components $\mu(t)=\mu_{1}(t)+\mu_{2}(t)$.

The autocorrelation function (ACF) $r_{\mu \mu}(\tau)$ of the scattered component $\mu(t)=\mu_{1}(t)+j \mu_{2}(t)$ can be obtained by taking the inverse Fourier transform of the Jakes power spectral density of Eq.(5):

$$
r_{\mu \mu}(\tau)=2 \sigma_{0}^{2} J_{0}\left(2 \pi f_{m} \tau\right)
$$

where $J_{0}(\cdot)$ is the zeroth-order Bessel function of the first kind.

\section{FREQUENCY ZERO-APPENDING FOR TIME INTERPOLATION}

Zero-padding is a well-known topic in signal analysis, normally associated with upsampling or unifying signals' lengths in circular convolution.

In this work we use zero-padding in the frequency domain for the purpose of increasing the resolution in
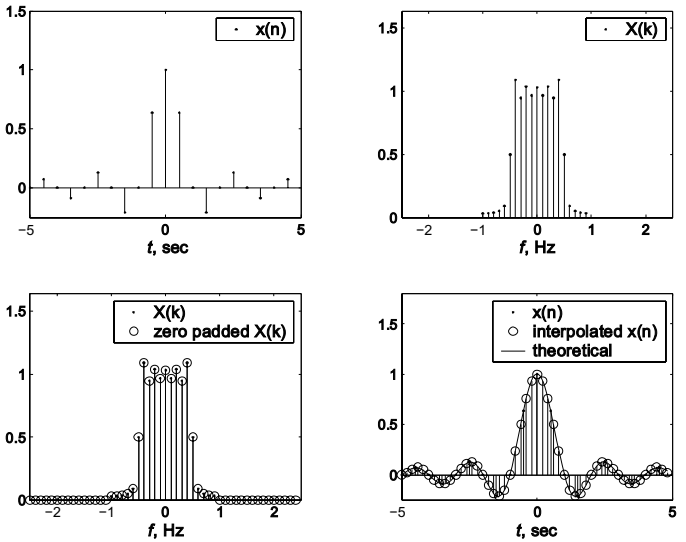

Fig. 2. Zero-appending $X(k)$ to provide finer interpolation for a time sequence $x(n)$ with duration $2 L=10$ s and sampling period $T_{s}=0.5 \mathrm{~s}$. After zero-appending, time duration remains unchanged, but the new sampling period is $T_{s}=0.2 \mathrm{~s}$, thus a better resolution is achieved for $x(n)$.

the time domain. This approach is defined by simply appending a number of zeros to both ends of the spectrum, hence we use the name zero-appending. Based on the duality property of the Fourier Transform, we can state that zero-appending in the frequency domain corresponds to ideal interpolation in the time domain. We demonstrate this theorem using a simple example. The sequence $x(n)$ is obtained by sampling a sinc function $x(t)=\operatorname{sinc}(t / T)$ with $T=1 \mathrm{~s}, T_{s}=0.5 \mathrm{~s}$, and the total time duration is from $-L=5 \mathrm{~s}$ to $L=5 \mathrm{~s}$, $N=2 L / T_{s}$, therefore $N=20$, that is, both $x(n)$ and its DFT $X(k)$ are 20 point sequence. We exploit again the time-domain/frequency-domain duality of DFT, that is, the time duration of the signal in time domain will determine the frequency resolution in frequency domain, while the frequency span in frequency domain will determine the time resolution in time domain. We append a total of $M-N$ zeros to the spectrum, divided into two halves, one half on each end of the spectrum of $X(k)$. An IDFT is then performed on $X(k)$ to provide a finer interpolation in the time domain. Fig. 2 shows that a total of $M-N=30$ zeros are padded in the frequency domain to provide sufficient detail to yield a good picture of $x(n)$.

\section{DESIGN AND ANALYSIS OF THE RAYLEIGH FADING CHANNEL}

Smith demonstrated a computer simulator of Rayleigh fading channel in [3]. A detailed presentation of this method can also be found in [2]. This method uses a complex Gaussian random number generator to produce a line spectrum with complex weights in the positive frequency band. The maximum frequency component of the line spectrum is $f_{m}$. The negative frequency components are constructed by simply conjugating the complex Gaussian values obtained for the positive frequencies. The random valued line spectrum is then multiplied by a discrete frequency presentation 

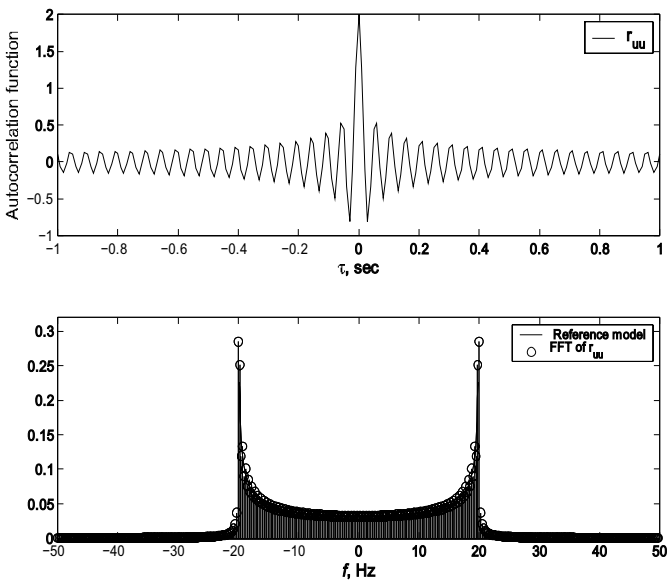

Fig. 3. The ACFs of the reference model and its FFT $\left(f_{m}=\right.$ $20 H z, \sigma_{0}^{2}=1, K=5, \Delta \tau=1 /\left(K f_{m}\right)=0.01 s, L=$ $2 s)$.

of $\sqrt{S_{u u}(f)}$ having the same number of points as the noise source. An IFFT is then performed on the resulting frequency domain signal to get two time series. The square root of the summed squared of the two time series is the Rayleigh fading signal with proper Doppler spread and time correlation. Note that this method requires a truncation of the spectrum because Equation (5) approaches infinity at the baseband edge. We present here a new method which avoids this truncation and gives better resolution by zero-appending. Moreover, we will show that this method promises greater mathematical tractability.

To implement this simulator, the following steps are used:

1. In Eq.(6), specify the power $\sigma_{0}^{2}$, maximal Doppler spectral $f_{m}$, and let $\Delta \tau=1 /\left(K f_{m}\right)$, here $K \geq 1$ is a constant. Define the range of $\tau$ from $-L$ to $L$. Compute the $N$ point $r_{\mu \mu}$.

2. To perform an FFT on $r_{\mu \mu}$ to get $N$ point $S_{\mu \mu}$.

3. Generate $N$ complex Gaussian random variables, normalize the total power to 1 .

4. Multiple the $N$ complex Gaussian random variable by the obtained Doppler spectrum $\sqrt{S_{\mu \mu}}$ from step 2 .

5. Perform an IFFT on the resulting frequency domain signal to obtain the $N$ point scattered component $u=u_{1}+j u_{2}$. The envelope of obtained $u$ is the simulated Rayleigh fading signal with the proper Doppler spread.

Fig.3 shows the ACF and its FFT with $f_{m}=20 \mathrm{~Hz}$, $\sigma_{0}^{2}=1, K=5, \Delta \tau=1 /\left(K f_{m}\right)=0.01 \mathrm{~s}, L=2 \mathrm{~s}$. Based on the discussion in the previous section, the parameters of the fading process $u$ can be easily calculated. The variance of $u, \sigma_{u}^{2}=(2 L) \sigma_{0}^{2}$, the duration of the fading signal $2 L=4 \mathrm{~s}$, and the time resolution of the fading signal $\Delta t=\Delta \tau=0.01 \mathrm{~s}$.

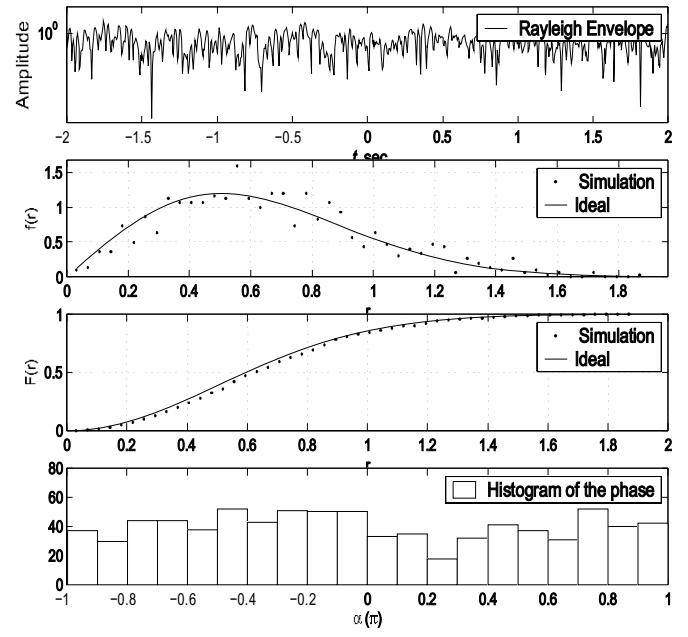

Fig. 4. The simulated fading envelope, its PDF and CDF, and the histogram of the fading phase $\left(f_{m}=20 \mathrm{~Hz}, \sigma_{0}^{2}=1\right.$, $\left.K=10, \Delta t=1 /\left(K f_{m}\right)=0.005 s, L=2 s\right)$.

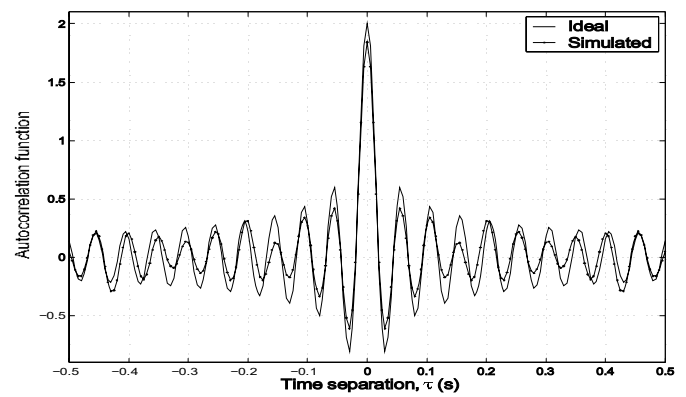

Fig. 5. The ACFs of the reference model and the simulation model $\left(f_{m}=20 \mathrm{~Hz}, \sigma_{0}^{2}=1, K=10, \Delta t=1 /\left(K f_{m}\right)=\right.$ $0.005 \mathrm{~s}, L=2 \mathrm{~s})$.

We now decide that the resolution is not satisfactory with $K=5$ and let $K=10$. The time resolution is thus doubled, $\Delta t=0.005 \mathrm{~s}$. The envelope of the simulated $u$ is shown in Fig. 4. The PDF of the envelope is Rayleigh distributed, and the phase of $u$ is uniformly distributed. The plot also shows the CDF of the envelope. The ACF of $u$ is plotted in Fig.5. Theoretical values are also plotted for the PDF, CDF and the ACF for the purpose of verification.

The advantages of this method are obvious. First, it is easy to implement. Second, the truncation of the spectrum is avoided. Third, a better resolution can be achieved by increasing the constant $K$, which means effectively appending more zeros to the Doppler spectrum. Moreover, the variance and the ACF of the simulated $u$ are simply related to the power $\sigma_{0}^{2}$ and the theoretical ACF specified in step 1: both are simply scaled down by the time duration $2 L$ defined in step 1. Based on the above discussion and the previous section, it is obvious that all the properties of the simulated Rayleigh fading signal can be specified in step 1, e.g., power, Doppler spread, time resolution, and time duration.

Apart from the probability density function, the 


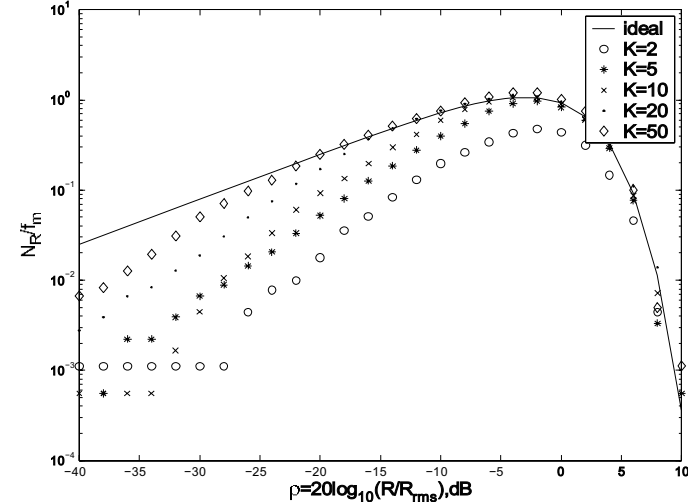

Fig. 6. Normalized level crossing rates of the envelopes. Simulation values obtained at $f_{m}=90 \mathrm{~Hz}, L=10 \mathrm{~s}$ for $K=2,5,10,20,50$.

cumulative distribution function, the autocorrelation function, and other statistical quantities that characterize the mobile fading channels like level crossing rate (LCR) and average duration of fades (ADF), are all important for the design of the channel simulator and mobile communication systems.

The level crossing rate, $N_{R}$, is defined as the expected rate at which the envelope crosses a specified signal level, $R$, in the positive direction. In general, it is given by [7]

$$
N_{R}=\int_{0}^{\infty} \dot{r} p(R, \dot{r}) d \dot{r}=\sqrt{2 \pi} f_{m} \rho e^{-\rho^{2}},
$$

where the dot indicates the time derivative and $p(R, \dot{r})$ is the joint density function of $r$ and $\dot{r}$ at $r=R$. And $\rho=R / R_{r m s}$ is the value of the specified level $R$, normalized to the local rms amplitude of the fading envelope. We simulate the $N_{R}$ for different $K$ at $f_{m}=90 \mathrm{~Hz}, L=10$ s and plot the results to compare with the ideal situation. From Fig. 6, the effect of zero-appending is significant. In this example, curves with $K>10$ give satisfactory results which are close enough to the ideal curve.

The average duration of fades, $\bar{\tau}$, is defined as the average period of time for which the received signal is below a specified level $R$. Let $\tau_{i}$ be the duration of the $i^{\text {th }}$ fade, then the average duration of fade for a total time interval of length $T$ is $\bar{\tau}=\sum \tau_{i} /\left(N_{R} T\right)$. Like level crossing rate $N_{R}$, the average duration of fade can be also expressed as a function of $\rho$ and $f_{m}$ as [7]

$$
\bar{\tau}=e^{\rho^{2}-1} / \rho f_{m} \sqrt{2 \pi}
$$

Fig. 7 shows the simulation of the average duration of fade for different $K$ at $f_{m}=90 \mathrm{~Hz}, L=10 \mathrm{~s}$ and the theoretical curve. Like the level crossing rate, the effect of zero-appending is significant. Again, curves with $K>10$ give satisfactory results which are close enough to the ideal curve. Simulation also shows that $K$ should not be arbitrarily large with the fixed time duration of $2 L$. In the example, overshoot is observed when $K>50$ for both the level crossing rate and the average duration of fades with $L=10$ s.

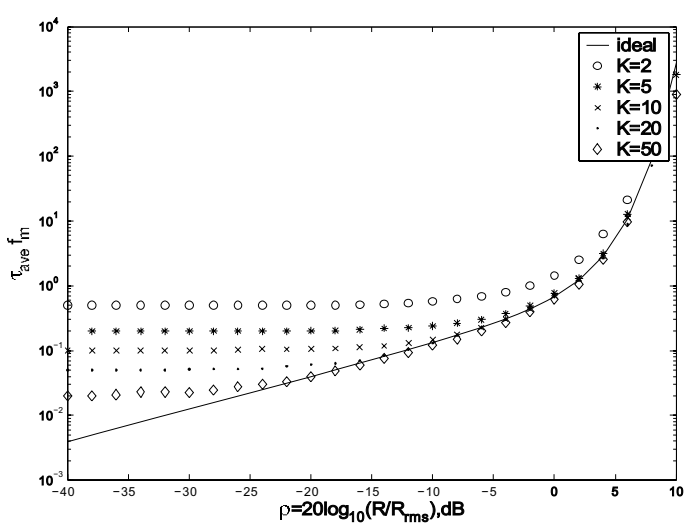

Fig. 7. Normalized durations of fade of the envelopes. Simulation values obtained at $f_{m}=90 \mathrm{~Hz}, L=10 \mathrm{~s}$ for $K=2,5,10,20,50$.

\section{CONCLUSIONS}

We presented a method to efficiently simulate Rayleigh fading channels. The significance of this method is its ease of implementation and greater mathematical tractability of the model parameters. Some important statistics especially the LCR and ADF of the model are investigated for the verification against the analytical model. Simulation results show that the channel simulator accurately reproduces all of the important statistical properties, such as the probability density function (PDF), autocorrelation (ACF), level crossing rate (LCR) and the average duration of fades (ADF).

\section{REFERENCES}

[1] M. Patzold and F. Laue, "Level-Crossing Rate and Average Duration of Fades of Deterministic Simulation Models for Rice Fading Channels," IEEE Trans. Vehicular Technology, vol. 48, no. 4, pp. 1121-1129, July. 1999.

[2] T. S. Rappaport, Wireless Communications, Principles and Practice, Prentice Hall, 2002, 2nd ed.

[3] J. I. Smith, "A Computer Generated Multipath Fading Simulation for Mobile Radio," IEEE Trans. Vehicular Technology, vol. 24, no. 3, pp. 39-40, Aug. 1975.

[4] S. S. Mahmoud, Z. M. Hussain, and P. O'Shea, "A Space-Time Model for Mobile Radio Channel With Hyperbolically Distributed Scatters," IEEE Antennas and Wireless Propagation Letters, vol. 1, pp. 211-214, 2002.

[5] R. H. Clarke, "A Statistical Theory of Mobile-Radio Reception," Bell Systems Technical Journal, vol. 47, pp. 957-1000, 1968.

[6] M. Patzold, Mobile Fading Channels, John Wiley and Sons, 2002.

[7] W. C. Jakes, Microwave Mobile Communications, Wiley, New York, 1974. 